\title{
Combining Cherenkov and scintillation detector observations with simulations to deduce the nature of high-energy radiation excesses during thunderstorms
}

\author{
Gregory S. Bowers, ${ }^{1,}$ William Blaine, ${ }^{1}$ Xuan-Min Shao, ${ }^{1}$ Brenda Dingus, ${ }^{1}$ David M. Smith, ${ }^{2}$ Michael Schneider, ${ }^{2}$ \\ Forest Martinez-McKinney, ${ }^{2}$ Michael P. McCarthy, ${ }^{3}$ Segev BenZvi, ${ }^{4}$ Lukas Nellen, ${ }^{5}$ and Nissim Fraija ${ }^{6}$ \\ ${ }^{1}$ Los Alamos National Laboratory, Los Alamos, New Mexico 87545, USA \\ ${ }^{2}$ University of California Santa Cruz, Santa Cruz, California 95064, USA \\ ${ }^{3}$ University of Washington, Seattle, Washington 98195, USA \\ ${ }^{4}$ University of Rochester, Rochester, New York 14604, USA \\ ${ }^{5}$ Instituto de Ciencias Nucleares, Universidad Nacional Autónoma de México, Mexico D.F. 04510, Mexico \\ ${ }^{6}$ Instituto de Astronoma, Universidad Nacional Autónoma de México, Mexico D.F. 04510, Mexico
}

(Received 20 June 2019; published 21 August 2019)

\begin{abstract}
We present co-observations of three strong count-rate enhancements associated with thunderstorms observed over 17 April 2015 to 23 September 2015 by the High Altitude Water Cherenkov (HAWC) array, and a suite of small scintillation detectors comprising the Gamma-ray Observations During Overhead Thunderstorms (GODOT) instrument. Because the HAWC array is most sensitive to ionizing radiation at high energies $(>100 \mathrm{MeV})$, and the small scintillation detectors are most sensitive to ionizing radiation at low energies (3-20 MeV), we investigate using the ratio of these detector counting rates variations to infer spectral characteristics of these enhancements, and understand the physics behind these thunderstorm accelerator mechanisms. We consider two extreme mechanisms that can produce these enhancements: a point source of relativistic runaway electron avalanches (RREA), and modification of the background cosmic-ray spectrum (MOS) from an electric field profile that is everywhere below the RREA threshold. We simulate the responses of HAWC and the GODOT $12.7 \mathrm{~cm} \times \oslash 12.7 \mathrm{~cm} \mathrm{NaI(Tl)} \mathrm{scintillator} \mathrm{and} \mathrm{show}$ that their ratio can discern between the two models, and that the observed thunderstorm rate enhancements are incompatible with the spectra from a point source of RREA, but consistent with our model of MOS for thunderstorm potentials within the range of -250 to $250 \mathrm{MV}$.
\end{abstract}

DOI: $10.1103 /$ PhysRevD.100.043021

\section{INTRODUCTION}

The electric field inside thunderstorms can cause significant variations in the background flux of high-energy radiation over timescales of seconds to tens of minutes, which have been observed from the ground [1-12], aircraft [13-15], and balloon [16]. These variations are attributed to the acceleration of background cosmic-ray secondaries [17] and the possible runaway and multiplication of electrons in the thunderstorm electric field.

For any field with finite extent, the energy spectra of cosmic-ray secondaries passing through a thunderstorm will be modified by the electric field. Termed modification

\footnotetext{
*gsbowers@lanl.gov
}

Published by the American Physical Society under the terms of the Creative Commons Attribution 4.0 International license. Further distribution of this work must maintain attribution to the author(s) and the published article's title, journal citation, and DOI. of spectrum (MOS) [17], this effect can produce variations in the count rates of ground based detectors [18].

When the electric field exceeds a certain strength called the break-even value, $E_{\mathrm{BE}}$, energetic electrons in the air will gain more energy than they lose to collisions and can run away to relativistic energies [19] up to $40 \mathrm{MeV}$. In air, $E_{\mathrm{BE}}=218 \mathrm{kV} / \mathrm{m} \times \rho(z) / \rho_{0}$, where $\rho(z)$ is the density of air at altitude $z$, and $\rho_{0}$ is the density of air at sea level [20]. For higher electric field strengths, it is possible for the runaway electrons to collide with molecules in the atmosphere and produce secondary energetic electrons through Møller scattering, which can themselves run away, and so produce relativistic runaway electron avalanches (RREA) [21]. RREA can occur in regions with field strengths above $E_{\text {RREA }}=284 \mathrm{kV} / \mathrm{m} \times \rho(z) / \rho_{0}$ [22-24] that extend over a suitable distance, comparable to the avalanche length, which is itself function of the altitude and field strength [25]. These runaway or RREA electrons can then produce bremsstrahlung emission in the atmosphere, creating gamma rays which can be observed at much greater distance than the electrons [1]. Long duration $\mathrm{x}$-ray and 
gamma-ray emission attributed to the bremsstrahlung of electrons produced by RREA are termed gamma-ray glows.

It is expected that count-rate variations associated with thunderstorm activity, also called thunderstorm ground enhancements (TGEs) [17], can be explained by a combination of MOS and RREA [18]. Since the energy spectra of the excess fluxes produced by these mechanisms can be very different, studying the spectral characteristics of TGEs can provide insight into thunderstorm characteristics [26,27].

Cosmic-ray observatories are valuable instruments for studying thunderstorm count-rate variations owing to their large sizes and ability to collect good statistics on individual events. The High Altitude Water Cherenkov (HAWC) gamma-ray observatory is the first Cherenkov-type instrument to report observations of thunderstorm ground enhancements. A limitation of HAWC is that it can report only the count rates of events and not the spectra. In this work we show that by combining count-rate observations from large Cherenkov-type detectors (HAWC) with observations from small, inexpensive scintillation detectors, the ratio of these detector variations along with simulations that model the detector responses can be used to infer properties of the event spectra.

Previous work has presented count-rate variations at HAWC associated with thunderstorm activity [28]. In this work we present three colocated observations of TGEs by HAWC and the Gamma-ray observations during overhead thunderstorms (GODOT) instrument, comprised of small scintillation detectors. We compare the observed detector ratios between HAWC and the GODOT $5 " \times 5 " \mathrm{NaI}$ scintillation detector during these TGEs to GEANT4 simulated detector ratios for extreme cases of MOS and RREA. Although the GODOT NaI detector can resolve spectral information well, its limited energy range for this deployment (below $11.7 \mathrm{MeV}$ ) and small physical size are not well suited to discern spectral differences between MOS and RREA, which exhibit significant differences above $40 \mathrm{MeV}$ where a large detector area is needed to collect good statistics. However, because HAWC is large and its counting rate is most sensitive to high energies $(\gtrsim 100 \mathrm{MeV})$, we show that the ratio of count rates from these detectors (which possess very different spectral responses) can distinguish between extreme cases of MOS and RREA, and that the observed TGEs are more consistent with our model of MOS than with RREA.

\section{INSTRUMENTS}

\section{A. HAWC}

The HAWC Gamma-ray Observatory is an array of large Cherenkov detector water tanks located at an altitude of $4.1 \mathrm{~km}$ above sea level in the mountains outside of Puebla, Mexico. The array consists of 300 water Cherenkov detectors (WCDs). Each WCD is comprised of a $7.3 \mathrm{~m}$ diameter and $5 \mathrm{~m}$ tall steel tank filled with purified water and containing 4 photomultiplier tubes (PMTs) mounted at the bottom: a large 10" PMT in the center surrounded by three smaller 8" PMTs. The PMTs are sensitive to Cherenkov light produced by ionizing particles that pass through the water in the tank [29]. The HAWC data are handled by a primary and secondary data acquisition (DAQ) system. In this study we use data from the secondary DAQ, called the scaler rate, which records the number of pulses within $100 \mathrm{~ms}$ windows. A more detailed description of the raw HAWC scaler data and processing is discussed in the Appendix.

\section{B. GODOT}

The GODOT instrument detector is a suite of scintillation detectors designed to measure the emission of energetic particles associated with lighting [30]. The instrument consists of three scintillation detectors: a $12.7 \mathrm{~cm} \times$ $\oslash 12.7 \mathrm{~cm} \mathrm{NaI}(\mathrm{Tl})$; a $12.7 \mathrm{~cm} \times \oslash 12.7 \mathrm{~cm}$ plastic (termed $\mathrm{LgPl}$ ); and a $2.54 \mathrm{~cm} \times \oslash 2.54 \mathrm{~cm}$ plastic (termed $\mathrm{SmPl}$ ). The large detectors are situated beside each other, with the small plastic sitting on top of the two. Each scintillator is coupled to a PMT whose output is fed over coaxial cable to a Bridgeport eMorpho analog digital converter (ADC) that records the integrated PMT pulse output and its associated time stamp. To monitor for possible noise contamination from radio frequency (RF) coupling to the PMT system and coaxial cabling, there is an eMorpho connected over coax to an unloaded (containing no scintillator) copy of the small scintillation detector under high voltage. To monitor for RF coupling to the coaxial cabling, there is an eMorpho connected to a 6" $50 \mathrm{ohm}$ terminated coaxial cable. The entire instrument is contained within an aluminum box that has been shielded from RF, and ruggedized for outdoor deployment and transportation.

The GODOT detectors can measure gamma rays down to $\sim 100 \mathrm{keV}$. To remove radon contamination from our measurements, we only consider counts in the GODOT detectors with energies above $3 \mathrm{MeV}$. We note that this is overly conservative with respect to excluding rate variations from rainout and washout, as the most abundant rainout progeny do not have significant gamma-ray emissions above 2.44 MeV [1]. Because the strongest natural terrestrial background gamma-ray emission is $2.614 \mathrm{MeV}$ (from 208Tl), the GODOT background counting rates above $3 \mathrm{MeV}$ can be considered entirely due to flux from the cosmic-ray background. More details of the raw GODOT data and processing are discussed in the Appendix.

\section{TGE OBSERVATIONS}

The HAWC site generally sees thunderstorm activity for 6 months out of the year [28], during spring and summer, with peak activity towards the end of summer, and some activity out to early fall.

From 2015, April 17th to September 21st, GODOT was installed in the counting house situated in the center of the 


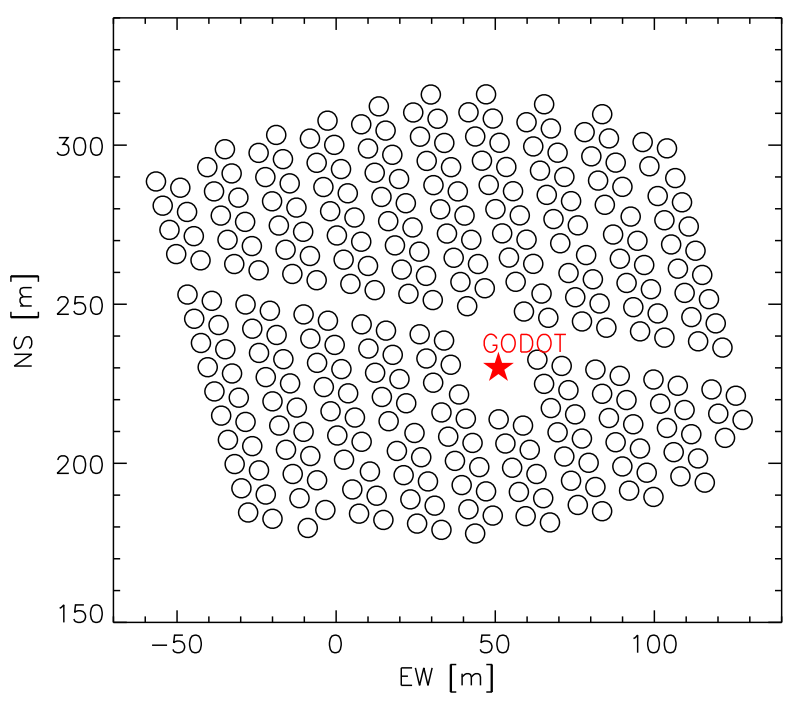

FIG. 1. The 300 HAWC WCDs indicated by circles and the relative location of the GODOT detector (red star) during 2015, April 17th to September 21st.

HAWC array, shown in Fig. 1. During this time there were three strong count-rate enhancements, or TGEs, identified in GODOT's NaI detector data for energies $>3 \mathrm{MeV}$. These events were flagged by identifying significant excess rates above $3 \mathrm{MeV}$ in spectrograms of the GODOT NaI scintillator data. For the TGE observed on 2015, May 3rd (TGE 150503), the peak count rate in the GODOT NaI corresponded to a $9.1 \sigma$ excess above background. Two TGEs observed by the GODOT NaI on 2015, July 15th at 21:10 UTC and 22:40 UTC (TGE 150715a,b) had peak rates at the $7.4 \sigma$ and $5.6 \sigma$ significance level respectively. For each of the three TGEs identified in the GODOT NaI spectrogram data, there was a coincident variation in the HAWC scaler rates. The 150503 TGE corresponded to the highest count-rate variation, and is shown in the left-most 4-panel column in Fig. 2. The two smaller 150715 TGEs are shown in the center and right-most 4-panel columns in Fig. 2.

To achieve good timing resolution and counting statistics, the rates for TGE 150503 were binned at $8 \mathrm{~s}$ intervals, and TGE $150715 \mathrm{a}$ and TGE $150715 \mathrm{~b}$ used $64 \mathrm{~s}$ intervals. For all HAWC and GODOT NaI and LgPl data in Fig. 2 we additionally show the variation, $\Delta R / R_{0}$, defined to be

$$
\frac{\Delta R}{R_{0}}=\frac{R-R_{0}}{R_{0}}
$$

where $R_{0}$ is the detector's background counting rate, and $R$ is any nonbackground counting rate of interest.
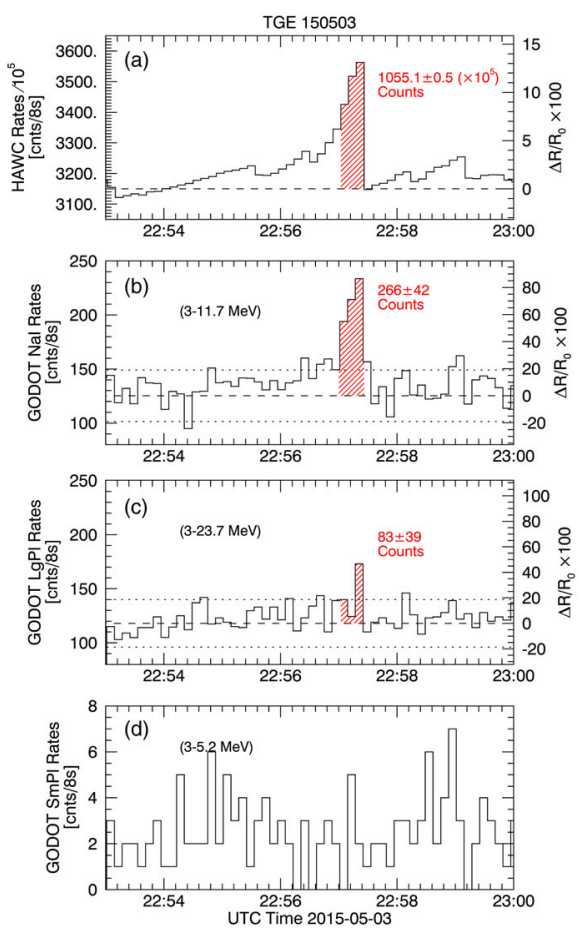
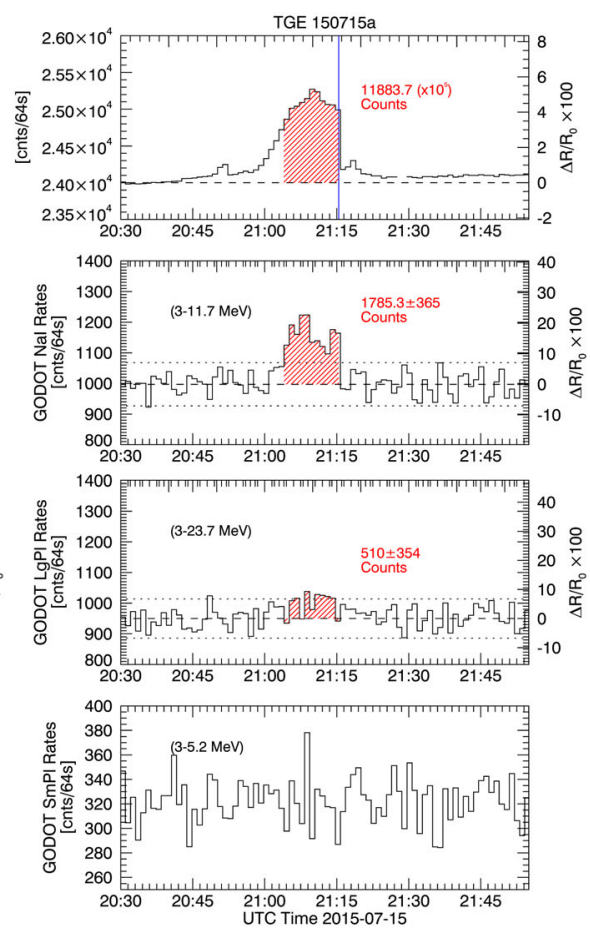
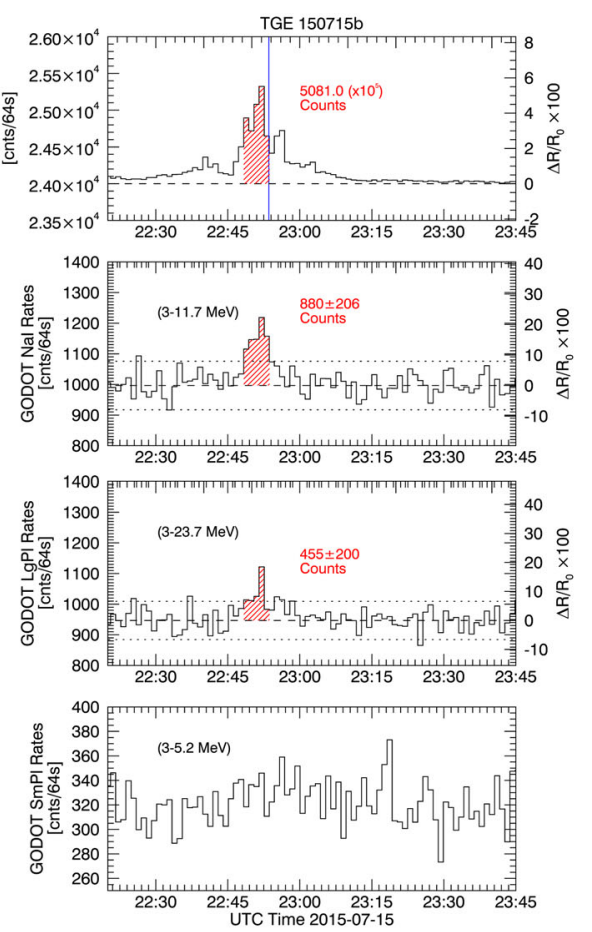

FIG. 2. Thunderstorm ground enhancements co-observed by (a) HAWC and the three GODOT detectors, (b) $5 \times 5$ NaI, (c) $5 \times 5$ plastic ( $\mathrm{LgPl}$ ), (d) $1 \times 1$ plastic (SmPl) for the dates of 2015 May 15, (left column), July 15th at 21:10 UTC (middle column), and July 15th at 22:40 UTC (right column). For each detector (except SmPl) the background rate is shown (dashed line) as well as the $2 \sigma$ tolerance interval (fine dashed). The shaded regions show excess counts (background subtracted total) attributed to the thunderstorm variation, with the total number of counts in the shaded region indicated in red. Blue vertical lines in the HAWC data show nearby lightning flashes registered by WWLLN. 
The observed variations consist of gradual increases in the count rates over a 2 to 10 minute duration, which abruptly return to the quiet-time background (within a $100 \mathrm{~ms}$ interval observed by HAWC), indicating the glow was terminated by a lightning process, consistent with previous observations of so-called "lightning terminated gamma-ray glows" [6,31]. Although changes in atmospheric pressure and density can cause count-rate variations, the sudden termination of these enhancements clearly show an association with similarly sudden changes in the thunderstorm electric field.

During TGE 150503 there were no World Wide Lightning Location Network (WWLLN) registered flashes during the TGE. However WWLLN did locate 11 strokes between 10 and $20 \mathrm{~km}$ from HAWC during an 18 minute interval centered on 22:56:30, which implies a nearby active thunderstorm cell. During TGE 150715a and b, WWLLN did register nearby lightning coincident with the "shutoff" (return to background rates) of the TGEs, at times indicated in Fig. 2.

The relative response of the three GODOT detectors are consistent with real particle observations. That is, while the $\mathrm{NaI}$ and large plastic $(\mathrm{LgPl})$ scintillators have the same volumes, the $\mathrm{NaI}$ is $\sim 3$ times more sensitive to gamma rays than the large plastic because of its higher gamma-ray cross section, which is observed in the higher $\mathrm{NaI}$ count rates compared to $\mathrm{LgPl}$. The lack of any discernible variation in the small plastic detector's count rate is consistent with the fact that it is $\sim 100$ times less sensitive to gamma rays as compared to the large plastic based on difference in scintillator volumes $(1: 125)$. While we do not consider the $\mathrm{LgPl}$ and $\mathrm{SmPl}$ detector data further in this study, they are presented to demonstrate a consistent physical instrument response for these enhancements. No RFI was detected on the GODOT noise monitoring channels.

In Fig. 2(a), there appear fluctuations outside of the shaded region which do not resemble the Gaussian fluctuations observed during fair weather. The fluctuations consist of gradual rises in the count rate (on the order of a few percent change in variation) over 10s of seconds and then abrupt drops. The largest fluctuation outside the red region in Fig. 2(a) appears at 22:59 and is associated with a marginal peak in the NaI count rate in Fig. 2(b). It is believed that these are fluctuations are faint TGEs, near the threshold of the NaI sensitivity. Although such "faint TGEs" are outside the scope of the current work, they demonstrate the usefulness of HAWC for future TGE studies.

\section{MODELING}

\section{A. Instrument response models}

Figure 3 shows the simulated effective areas for the HAWC and GODOT NaI detector. The effective area for the GODOT NaI is for detector counts in the energy range of $3-11.7 \mathrm{MeV}$ that is used in this study.

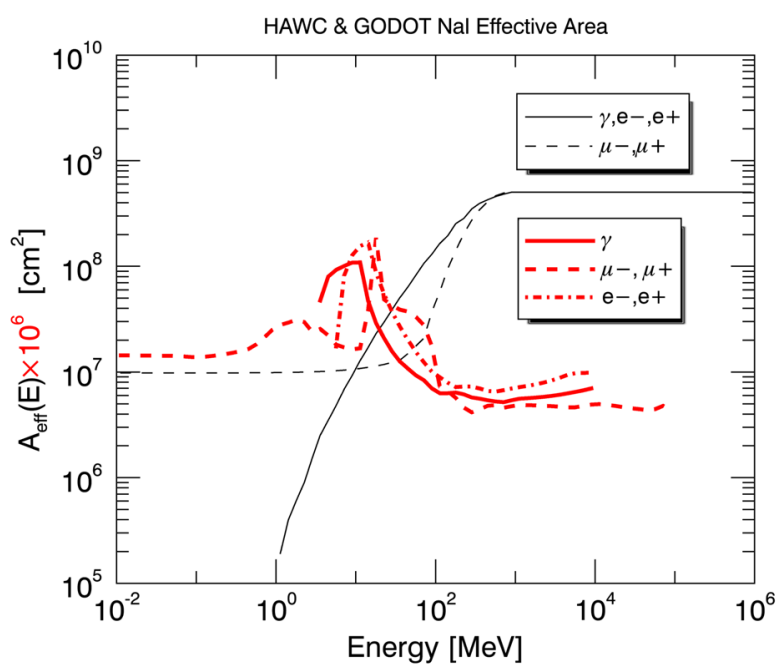

FIG. 3. HAWC and the GODOT NaI detector effective area for gammas, electrons, positrons and muons as a function of kinetic energy. HAWCs effective areas are indicated by the black lines, the GODOT NaI effective areas are indicated by the thick red lines. The NaI effective areas have been multiplied by $10^{6}$.

Given a differential flux of particles of type i, specified by $d \phi_{i} / d \mathcal{E}$, where $\phi$ is the flux, and $\mathcal{E}$ is the particles' kinetic energy, the counting rate in the detector from all particle species is determined from the detectors' effective areas by the relation

$$
R=\sum_{i} \int \frac{d \phi_{i}}{d \mathcal{E}} A_{i}(\mathcal{E}) d \mathcal{E}
$$

As shown in Fig. 3, for a given particle flux, HAWC is most sensitive to the high-energy end of the spectrum $(\gtrsim 100 \mathrm{MeV})$, and the GODOT NaI detector is most sensitive to particles within the range of $\sim 3-20 \mathrm{MeV}$.

Details of the simulations used to calculate the detector effective areas for HAWC and GODOT as well as simulations of the GODOT NaI spectra are presented in the Supplemental Material [32].

\section{B. TGE modeling overview}

To model thunderstorm count-rate variations and isolate the distinct spectra produced by RREA and MOS, we simulate extreme cases of both mechanisms using GEANT4, a Monte-Carlo simulation toolkit to model the passage of particles through matter [33-35]. For this work we used the FTFP_BERT_HP_LIV physics list, which uses the Fritiof string theory and Bertini intranuclear cascade models for hadronic interactions, and the Livermore low-energy electromagnetic suite of interaction models. HP specifies high precision models for low energy neutrons. Recent work by Sarria et al. [36] has shown that the reference electromagnetic physics lists in GEANT4 must be modified to accurately model the RREA process, which was done for 
this work, although was likely not necessary as we do not simulate particle interactions in fields above the RREA threshold, and we do not simulate RREA directly, but instead use the expected gamma-ray output from an RREA development.

We model extreme MOS by simulating the passage of the cosmic-ray background through an electric field profile that is everywhere below the RREA threshold. We model extreme RREA by assuming a point source region of gamma rays that follow the energy distribution of bremsstrahlung gammas produced by RREA electrons.

We are interested in simulating the TGE count-rate variations in the HAWC and GODOT NaI detectors for each mechanism, denoted by $\Delta R_{\mathrm{MOS}} / R_{0}$ and $\Delta R_{\mathrm{RREA}} / R_{0}$. We then consider the ratio of detector variations

$$
\frac{\left(\Delta R / R_{0}\right)_{\mathrm{HAWC}}}{\left(\Delta R / R_{0}\right)_{\mathrm{GODOT}}}
$$

which can be simulated for MOS and RREA and then compared to observations. From the effective areas of the HAWC and GODOT NaI detectors in Fig. 3, it is expected that between two TGE mechanisms, the one with the softer spectra will have the lower ratio.

To model MOS, we simulate the propagation of cosmicray secondaries through an atmosphere with different electric field conditions. We first simulate fair-weather (no-field) background rate, $R_{\mathrm{sim}, 0}$, by convolving the simulated fair-weather ground flux with the instrument effective areas according to Eq. (2). We then simulate the ground flux resulting from the presence of a nonzero electric field region that is everywhere below the RREA threshold, and calculate the MOS rate, $R_{\mathrm{MOS}}$. The excess rate due to MOS is $\Delta R_{\mathrm{MOS}}=R_{\mathrm{MOS}}-R_{\mathrm{sim}, 0}$, and the simulated MOS variation is defined by

$$
\frac{\Delta R_{\mathrm{MOS}}}{R_{0}}=\frac{R_{\mathrm{MOS}}-R_{\mathrm{sim}, 0}}{R_{\mathrm{sim}, 0}} .
$$

To model RREA, we use a point source of bremsstrahlung RREA gamma rays that are input into a mass model of our atmosphere at various altitudes. All secondary and albedo particles crossing a collection surface at $4.1 \mathrm{~km}$ are recorded. This flux is convolved with the instrument effective areas to produce the RREA rate, $R_{\text {RREA }}$. Since our model of RREA is independent of the fair-weather background, the excess rate due to RREA is the simulated RREA rate, or $\Delta R_{\mathrm{RREA}}=R_{\mathrm{RREA}}$, and the simulated RREA variation is

$$
\frac{\Delta R_{\mathrm{RREA}}}{R_{0}}=\frac{R_{\mathrm{RREA}}}{R_{\mathrm{obs}, 0}} .
$$

Note that unlike the variation defined for MOS in Eq. (4), our simulated RREA flux contains no contribution from background. Since our RREA simulation cannot model background, we used the observed background rate to define the variation (it would be acceptable to use the simulated MOS background as well).

\section{MOS model}

To model the cosmic-ray background above HAWC, we use the Excel-based Program for Calculating Atmospheric Cosmic-ray Spectrum (EXPACS) [37,38], previously used in other TGE studies $[17,26,27]$. Here we use EXPACS to specify the energy spectrum and angular distribution for gamma rays, electrons, positrons, muons and neutrons, which are input into a mass model of the atmosphere at $8 \mathrm{~km}$ with a vertical extent from 0 to $10 \mathrm{~km}$. All secondary and albedo particles crossing a collection surface at $4.1 \mathrm{~km}$ are recorded. Details of our cosmic-ray background modeling can be found in the Supplemental Material [32].

To simulate the most extreme case of modification of spectra, we consider a "maximal MOS" electric field profile, which is defined to be a unipolar electric field profile that varies with altitude according to

$$
E_{\mathrm{MOS}}(z)=E_{0} \rho(z) / \rho_{0}
$$

where $E_{0}$ is the sea level equivalent electric field, and the associated maximal MOS potential $U_{\text {MOS }}$ is defined by

$$
U_{\mathrm{MOS}}(z)=-\int_{z_{1}}^{z 2} E_{\mathrm{MOS}} \mathrm{d} z
$$

For this study we have assumed an exponential atmospheric density profile $\rho(z) / \rho_{0}=\exp (-z / h)$ with a scale height of $h=8.3 \mathrm{~km}$, following the US standard atmosphere model [39]. For our simulations the vertical extent of the electric field region is from $z_{1}=4.1 \mathrm{~km}$ (just above HAWC) to $z_{2}=8 \mathrm{~km}$. While we did not observe the thunderstorm structure for these events, recent observations at HAWC using the broadband RF interferometric mapping and polarization (BIMAP) instrument [40] have located cloud-to-ground lightning initiation at $8 \mathrm{~km}$ altitude, or $\approx 4 \mathrm{~km}$ above HAWC.

The E-field model in Eq. (6) guarantees that the E-field in the whole atmosphere is below the RREA threshold for $E_{0}<E_{\mathrm{RREA}}$, and that any count-rate enhancement will be entirely due to MOS, with no RREA production. This is not guaranteed to be the case for more realistic uniform electric field profiles, which over a sufficient vertical extent may contain a mix of MOS and RREA regions. It is emphasized that we present no physical justification for choosing an atmospheric electric field profile to scale with the atmospheric density, but that it is only to create a model that isolates the MOS spectra for study. Details of our MOS simulations are in the Supplemental Material [32].

The simulated ratio of detector variations from MOS is shown in Fig. 4(a), for values of $E_{\mathrm{MOS}}$ ranging from 

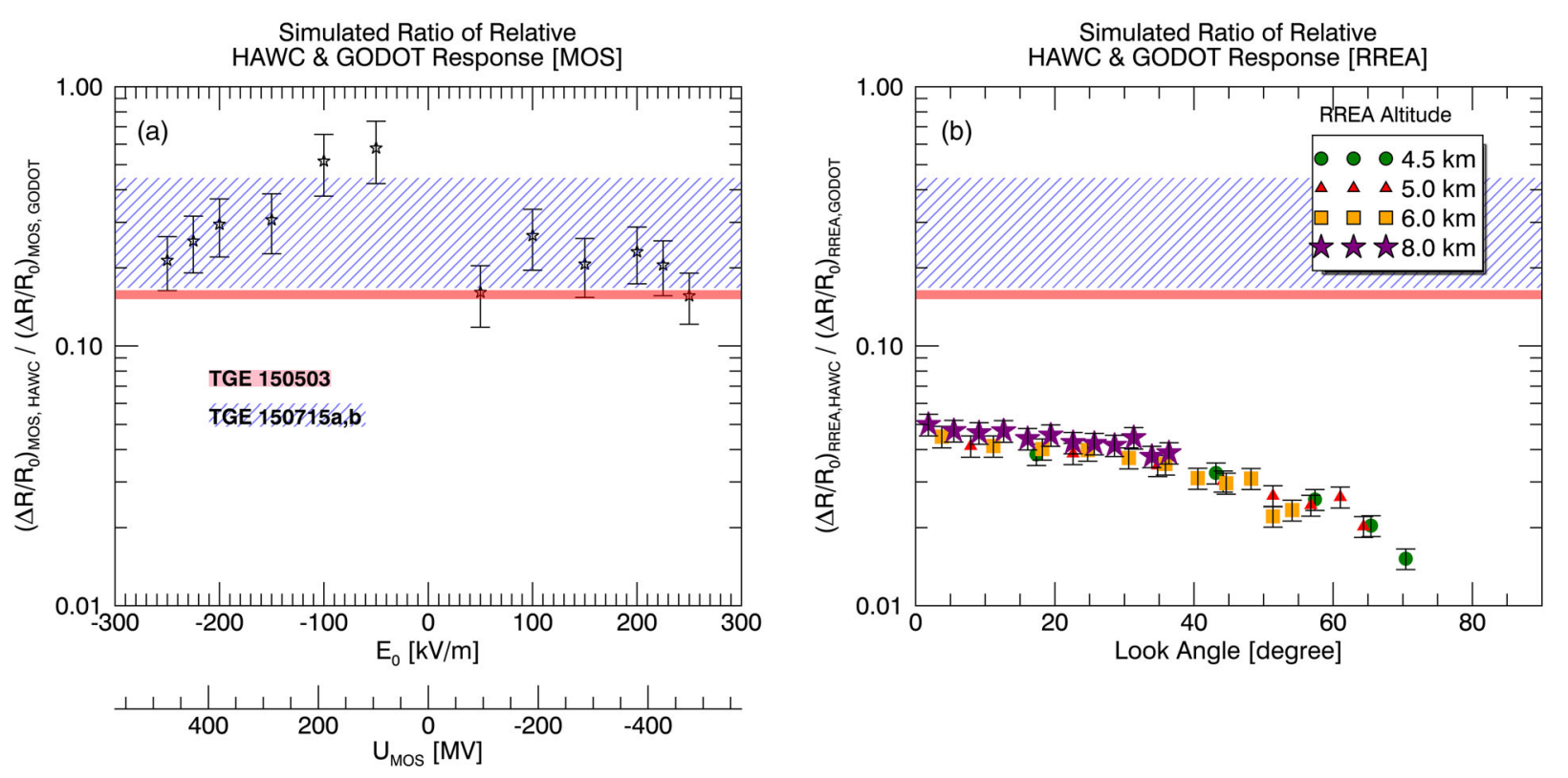

FIG. 4. Simulated ratio of detector variations for MOS and RREA. (a) Simulated ratio of HAWC and GODOT NaI detector variations for MOS, as a function of the sea level equivalent electric field, $E_{0}$, that parametrizes the electric field profile, as well as the total MOS potential, $U_{\text {MOS }}$. (b) Simulated ratio of HAWC and GODOT detector variations for a point source of RREA, as a function of zenith viewing angle to the source, for various source altitudes. The range of observed detector variations from each time bin in the shaded regions in Fig. 2, $\left(\Delta R / R_{0}\right)_{\mathrm{HAWC}, \mathrm{obs}} /\left(\Delta R / R_{0}\right)_{\mathrm{GODOT}, \mathrm{obs}}$, for TGE 150503 and TGE $150715 \mathrm{a}$, b are indicated by red and blue band respectively. The error bars indicate uncertainties from Poisson statistics (see Supplemental Material [32]).

$-250 \mathrm{kV} / \mathrm{m}$ to $+250 \mathrm{kV} / \mathrm{m}$. Simulated detector variations from MOS for individual detectors are presented in the Supplemental Material [32]. The ratio of detector variations is asymmetric with respect to the total MOS potential. For slightly positive potentials (corresponding to a negative overhead charge layer), the ratio is high $(\sim 0.5)$, and for slightly negative potentials, the ratio is lower $(\sim 0.2)$. This is due to a combination of four effects: (1) the low-energy charged particle background is asymmetric (more $e-$ than $e+)$; (2) the high-energy charged particle background is approximately symmetric (approximately the same number of $\mu^{-}$and $\mu^{+}$); (3) the GODOT NaI is most sensitive to low energy particles; (4) the HAWC array is most sensitive to high-energy particles. From (1) and (2) it follows that for small positive and negative potentials of equal magnitude, the variation in the GODOT NaI rate will be greater for a negative overhead charge layer (which shields the low energy electrons above the thunderstorm from reaching the ground) than for a positive overhead charge layer (which shields the low energy positrons above the thunderstorm from reaching the ground), and therefore the GODOT variation will be asymmetric with respect to field polarity. From (3) and (4) it follows that the variation in the HAWC rate is more symmetric since the $\mu^{-}$and $\mu^{+}$fluxes are comparable. The net effect is that the ratio of detector variations defined in Eq. (3) will be larger for slightly negative electric fields than for slightly positive electric fields (of the same magnitude), which is observed in the simulated output. It is noted that as the magnitude of the
MOS increases, the ratio tends to decrease, which is consistent with previous work noting that as the MOS field increases, the spectra tends to soften [27].

\section{RREA point source model}

We consider the extreme case of a pointlike source of RREA, in which avalanche production occurs within an infinitely small electric field region and does not produce modification of spectra (corresponding to 0 electric field). To model pointlike RREA, we use an analytic RREA bremsstrahlung photon spectra, typically used to model the spectra of electrons at the end of an RREA region [20,30], given by

$$
f_{\text {RREA }, \gamma}(\mathcal{E}) \sim \exp (-\mathcal{E} / 6.5 \mathrm{MeV}) / \mathcal{E}
$$

where $\mathcal{E}$ is the photon energy. We use a $15.3^{\circ}$ half-angle Gaussian angular distribution from [41], and sample photons up to $40 \mathrm{MeV}$. To simulate the instrument response to RREA, we input this photon spectra as a pointlike source located at various altitudes $(h=4.1,5,6$, $8 \mathrm{~km}$ ) into a mass model of our atmosphere and record the resulting flux (including albedo) at $4.1 \mathrm{~km}$, the altitude of HAWC. We sample the flux within a $250 \mathrm{~m}$ wide annulus centered around the radial offset $r$ from the nadir of the input source, representing the observed RREA flux from a zenith viewing angle $\theta=\arctan (r / h)$. This flux is then input into the instrument response models, and the total 
variation is calculated as a function of source altitude and viewing angle.

The simulated ratio of detector responses from a point source of RREA is shown in Fig. 4(b). Simulated detector responses from a point source of RREA are presented in the Supplemental Material [32]. The ratio is seen to be insensitive to altitude, but decreases with increasing zenith observation angle, corresponding to an increase in the radial offset from the nadir of the RREA source. This is because as the detectors move away from a downward beam of RREA, the observed spectra softens due to Compton scattering. Had we considered an extended RREA source instead of a point source, we would expect less softening away from the nadir of the center of the source region, but overall a similar picture.

\section{TGE OBSERVATIONS AND MODELING COMPARISON}

The observed detector ratios for the three TGEs shown in Fig. 2 are compared to the simulated detector ratios for MOS and a RREA point source in Fig. 4. The horizontal colored bands indicate the range of observed ratios from TGEs 150503 and 150715a,b. The observed range of variations for TGE $150715 \mathrm{a}$ and $150715 \mathrm{~b}$ are very similar. The three co-observed TGE detector variations are more consistent with the simulated MOS variations than the point-source RREA variation.

\section{DISCUSSION}

Both models used to model thunderstorm ground enhancements, pointlike RREA, and extreme MOS, are likely unphysical. For example, a source region of RREA that does not produce MOS seems unreasonable, because any finite electric field region that would produce RREA would also likely produce significant MOS. Additionally, the total simulated MOS potential that can reproduce the magnitude of the observed detector variations in HAWC and GODOT for TGE 150503 is on the order of $\left|U_{\text {MOS }}\right|=100-275 \mathrm{MV}$, which is close to the limit of maximum observed thunderstorm potentials $(\approx 130 \mathrm{MV})$ [42]. Recent work has suggested that the thunderstorm potential can reach as high as $1.4 \mathrm{GV}$ [43], but this assumes electric field strengths that exceed the RREA threshold, and whose extent would likely produce large currents from RREA feedback $[23,44]$ that would quickly collapse such a potential. In spite of our assumptions, these simulations show that the ratio of counts from detectors with different effective areas can be useful in discerning spectral differences. In this case, the ratio can easily distinguish between the hard spectra of MOS from extended electric field regions below the RREA threshold, and the soft spectra of point source RREA.

The HAWC and GODOT NaI detector ratios observed during the three TGEs shown in Fig. 2 are more comparable to the simulated detector ratios for MOS than for RREA (Fig. 4). It is seen that the detector ratios during the stronger TGE 150503 are smaller than the detector ratios for the weaker TGE 150715a and b, indicating the TGE spectra for this event was softer, either because of higher MOS potentials, or from the additional presence of RREA.

\section{CONCLUSIONS}

(1) The HAWC observatory is capable of observing TGEs. We have presented the three strongest TGEs co-observed by HAWC and GODOT during 17 April 2015 to 23 September 2015. It is possible that HAWC is sensitive to weak TGEs that cannot be observed by small suites of scintillation detectors, such as those typically deployed to observe energetic emissions from lightning. An analysis of these weaker TGEs may provide important insight into the thunderstorm charging process.

(2) We have shown that the ratio of count-rate variations from detectors with different effective areas can be useful in discerning spectral differences in TGEs.

(3) The co-observation of three TGEs at HAWC are inconsistent with production from a point-source region of RREA. They are most consistent with production by MOS. It is possible (and probably likely) that these variations were caused by a TGE mechanism that was some combination of MOS and RREA, but without further details of the specific thunderstorm charge structure, it is not possible to further constrain the TGE mechanisms.

Comparisons of TGE observations with simulations of TGE mechanisms are limited by knowledge of the likely thunderstorm charge structure. Future observations that can combine TGE spectral observations with simultaneous observations of thunderstorm charge structure can provide new insights into the nature of the TGE mechanism and thunderstorm characteristics like in-situ electric field strengths and charging dynamics.

\section{ACKNOWLEDGMENTS}

We would like to acknowledge the Domestic Nuclear Detection Office under competitively awarded Contract No. IAA: HSHQDC-16-X-00088. The views and conclusions contained in this document are those of the authors and should not be interpreted as necessarily representing the official policies, either expressed or implied, of the U.S. Department of Homeland Security or the Government. This work was supported by Laboratory Directed Research and Development project 20170179ER in Los Alamos National Laboratory. The authors wish to thank the World Wide Lightning Location Network (http://wwlln.net), a collaboration among over 50 universities and institutions, for providing the lightning location data used in this paper. We acknowledge the support from: the U.S. National 
Science Foundation (NSF) the U.S. Department of Energy Office of High-Energy Physics; the Laboratory Directed Research and Development (LDRD) program of Los Alamos National Laboratory; Consejo Nacional de Ciencia y Tecnología (CONACyT), México (Grants No. 271051, No. 232656, No. 260378, No. 179588, No. 239762, No. 254964, No. 271737, No. 258865, No. 243290, No. 132197, No. 281653) (Cátedras 873, 1563, 341), Laboratorio Nacional HAWC de rayos gamma; L'OREAL Fellowship for Women in Science 2014; Red HAWC, México; DGAPA-UNAM (Grants No. AG100317, No. IN111315, No. IN111716-3, No. IA102715, No. IN109916, No. IA102019, No. IN112218); VIEPBUAP; PIFI (Programa Integral de Fortalecimiento Institucional) 2012, 2013, PRO-FOCIE (Programa de Fortalecimiento de la Calidad en Instituciones Educativas) 2014, 2015; the University of Wisconsin Alumni Research Foundation; the Institute of Geophysics, Planetary Physics, and Signatures at Los Alamos National Laboratory; Polish Science Centre Grant No. DEC-2014/13/B/ST9/945, No. DEC-2017/27/ B/ST9/02272; Coordinación de la Investigación Científica de la Universidad Michoacana; Royal Society-Newton Advanced Fellowship 180385. Thanks to Scott Delay, Luciano Díaz and Eduardo Murrieta for technical support.

\section{APPENDIX: RAW DATA AND DATA PROCESSING}

\section{HAWC Data}

Recent work has looked at counting rate contamination from Rn-222 [45]. During rainfall the low-energy background gamma-ray intensity can increase due to the concentration at the ground of radioactive isotopes from naturally occurring radon progeny that have been scavenged out of the atmosphere by rain drops. The most significant of these progeny are ${ }^{214} \mathrm{~Pb}$ and ${ }^{214} \mathrm{Bi}$ with half-lives of 26.8 and 20 minutes respectively, whose decays are accompanied by gamma-ray emissions. HAWC's effective area for gamma rays at these energies are very low (zero below $\sim 1 \mathrm{MeV}$ ), and it is assumed that contamination in the scaler counting rate from radon is negligible. We believe this is reasonable since general signatures of background-rate increases coincident with rainfall onset, or background-rate decay coincident with rainfall end are not observed, as would be expected if radon contamination were present.

It has been found that the HAWC array is susceptible to $\mathrm{RF}$ interference which can produce false counts in the data. During periods of intense electromagnetic activity the main DAQ is unstable and restarts frequently making the HAWC scaler counts generally unavailable during overhead thunderstorm activity. The secondary DAQ is stable but also susceptible to RF interference. This interference has been studied and found to be responsible for two types of artifacts that can be identified in the HAWC scaler rate data:
(1) impulsive rate spikes, or "noise bursts," with durations of a few ms, coincident with and proportional in both duration and intensity to the RF power generated by nearby lightning discharges as characterized by the co-located BIMAP instrument [40], (2) fast rise and exponential-like cecay (FRED) events, consisting of certain PMT rates jumping to very high rates (near or at the saturation rate) within $100 \mathrm{~ms}$, and then decaying over variable time scales from minutes to hours.

While the causes for this interference is not completely understood, a procedure was developed to select a subsample of the HAWC PMTs that did not exhibit FRED-like interference within 30 minutes of an observed scaler rate enhancement of interest, and a $2.5 \mathrm{~s}$ median smoothing filter was applied to remove the impulsive rate spikes which account for less than $3 \%$ of the count rate. By this criteria, of the 1044 PMTs that were operating at HAWC during these observations, for TGE 150503, 67 PMTs were selected as being "FRED-free," consisting of 32 8" PMTs $\left(N_{8}\right)$, and 3510 " PMTs $\left(N_{10}\right)$, while for TGE 150715a and b, 48 PMTs were selected, consisting of 36 8" PMTs and 12 10" PMTs.

For each TGE observation, the scaler rates from the FRED-free PMTs were combined and scaled up to an equivalent 1200 PMT rate to provide direct comparison with simulations. This scaling is done according to the formula

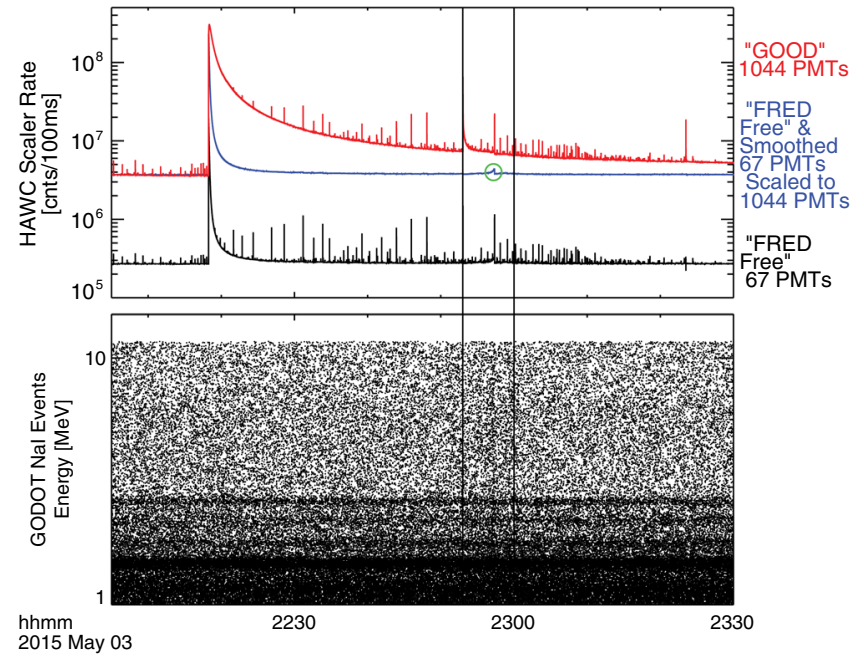

FIG. 5. Top: Raw and Processed HAWC Scaler rate data. (Red) combined count rate from 1044 "good" HAWC PMTs, showing a large FRED event near 2218 UTC. (Black) 67 FRED-free PMTs used for this event. FRED-free indicates no FRED event contamination within 30 minutes of our time interval of interest (vertical lines). (Blue) Combined 67 FRED-free PMT rates scaled up to equivalent 1044 PMT rate, smoothed with 2.5 second sliding median filter. Bottom: Raw GODOT NaI time-tagged event data. The 7-minute interval between the vertical lines corresponds to the data in Figs. 2(a) and 2(b). TGE 150503 seen by HAWC is indicated within the green circle. 


$$
R_{\mathrm{HAWC}}=\frac{900}{N_{8}} \sum^{N_{8}} R_{8, i}+\frac{300}{N_{10}} \sum^{N_{10}} R_{10, i}
$$

where $R_{[8,10], i}$ are the rates of $i$ th 8 " or 10 " PMTs classified as FRED-free.

Details of this selection procedure are shown for TGE 150503 in Fig. 5.

\section{GODOT Data}

The PMT output of each GODOT scintillator is monitored by a Bridgeport eMorpho ADC, which does pulse integration and time tags the result. It is assumed that the time integrated area of each PMT pulse is proportional to the total energy deposited in the scintillator crystal producing that pulse. The equivalent energy of each recorded pulse is calibrated to spectral features in background spectra accumulated over several hours, corresponding to the $2.64 \mathrm{MeV}^{208} \mathrm{Tl}$ and $1.45 \mathrm{MeV}^{40} \mathrm{~K}$ photopeaks. The calibrated time-tagged energy data is shown in Fig. 5, where each dot corresponds to a single ionizing particle interacting in the scintillator crystal.

The GODOT detector, which has operated for dozens of hours in active overhead thunderstorm conditions, has never exhibited signs of significant RF interference. During operation in the extreme RF environment near a Marx generator producing $1.5 \mathrm{MV}$ discharges over a $2 \mathrm{~m}$ air gap, GODOT experienced low levels of RF pickup, corresponding to contamination of the lowest energy channels $(<\mathrm{a}$ few hundred $\mathrm{keV}$ ) which are not considered in this study. None of the RF interference artifacts seen by HAWC have been observed by GODOT, and no interference was reported on the noise channels monitored by GODOT.
[1] D. M. Suszcynsky, R. Roussel-Dupre, and G. Shaw, J. Geophys. Res. 101, 23505 (1996).

[2] M. Brunetti, S. Cecchini, M. Galli, G. Giovannini, and A. Pagliarin, Geophys. Res. Lett. 27, 1599 (2000).

[3] A. P. Chubenko, V. P. Antonova, S. Y. Kryukov, V. V. Piskal, M. O. Ptitsyn, A. L. Shepetov, L. I. Vildanova, K. P. Zybin, and A. V. Gurevich, Phys. Lett. A 275, 90 (2000).

[4] T. Torii, M. Takeishi, and T. Hosono, J. Geophys. Res. 107, ACL 2-1 (2002).

[5] A. P. Chubenko, I. V. Amurina, V. P. Antonova, M. M. Kokobaev, S. V. Kryukov, R. A. Nam, N. M. Nesterova, V. V. Oskomov, V. V. Piscal, M. O. Ptitsyn, T. K. Sadykov, A. L. Shepetov, L. I. Vildanova, K. P. Zybin, and A. V. Gurevich, Phys. Lett. A 309, 90 (2003).

[6] V. V. Alexeenko, N. S. Khaerdinov, A. S. Lidvansky, and V. B. Petkov, Phys. Lett. A 301, 299 (2002).

[7] A. S. Lidvansky, J. Phys. G 29, 925 (2003).

[8] T. Torii, T. Nishijima, Z.-I. Kawasaki, and T. Sugita, Geophys. Res. Lett. 31, L05113 (2004).

[9] Y. Muraki, W. I. Axford, Y. Matsubara, K. Masuda, Y. Miyamoto, H. Menjyou, S. Sakakibara, T. Sako, T. Takami, and T. Yamada, Phys. Rev. D 69, 123010 (2004).

[10] T. Torii, T. Sugita, M. Kamogawa, Y. Watanabe, and K. Kusunoki, Geophys. Res. Lett. 38, L24801 (2011).

[11] X. X. Zhou, X. J. Wang, D. H. Huang, and H. Y. Jia, Astropart. Phys. 84, 107 (2016).

[12] B. Bartoli, P. Bernardini, X. J. Bi, Z. Cao, S. Catalanotti, S.Z. Chen, T. L. Chen, S.W. Cui, B.Z. Dai, and A. D’Amone, Phys. Rev. D 97, 042001 (2018).

[13] M. McCarthy and G. K. Parks, Geophys. Res. Lett. 12, 393 (1985).

[14] N. A. Kelley, D. M. Smith, J. R. Dwyer, M. Splitt, S. Lazarus, F. Martinez-McKinney, B. Hazelton, B. Grefenstette, A. Lowell, and H. K. Rassoul, Nat. Commun. 6, 7845 (2015).
[15] P. Kochkin, A. P. J. vanÂ Deursen, M. Marisaldi, A. Ursi, A. I. de $\hat{A}$ Boer, M. Bardet, C. Allasia, J.-F. Boissin, F. Flourens, and N. Østgaard, J. Geophys. Res. 122, 12 (2017).

[16] K. B. Eack, Rev. Sci. Instrum. 67, 2005 (1996).

[17] A. Chilingarian, B. Mailyan, and L. Vanyan, Atmos. Res. 114, 1 (2012).

[18] A. Chilingarian, G. Hovsepyan, and L. Kozliner, Phys. Rev. D 88, 073001 (2013).

[19] C. T. R. Wilson, Proc. Cambridge Philos. Soc. 22, 534 (1925).

[20] J. R. Dwyer, D. M. Smith, and S. A. Cummer, Space Sci. Rev. 173, 133 (2012).

[21] A. V. Gurevich, G. M. Milikh, and R. Roussel-Dupre, Phys. Lett. A 165, 463 (1992).

[22] E. M. D. Symbalisty, R. A. Roussel-Dupre, and V. A. Yukhimuk, IEEE Trans. Plasma Sci. 26, 1575 (1998).

[23] J. R. Dwyer, Geophys. Res. Lett. 30, 2055 (2003).

[24] L. P. Babich, E. N. Donskoy, R. I. Il'Kaev, I. M. Kutsyk, and R. A. Roussel-Dupre, Plasma Phys. Rep. 30, 616 (2004).

[25] L. M. Coleman and J. R. Dwyer, Geophys. Res. Lett. 33, L11810 (2006).

[26] A. Chilingarian, G. Hovsepyan, and L. Vanyan, Europhys. Lett. 106, 59001 (2014).

[27] E. S. Cramer, B. G. Mailyan, S. Celestin, and J. R. Dwyer, J. Geophys. Res. 122, 4763 (2017).

[28] A. Lara, G. B. de Raga, and O. Enriquez-Rivera (HAWC Collaboration), Int. Cosmic Ray Conf. 35, 80 (2017); Proc. Sci., ICRC2017 (2018) 080 [arXiv:1711.04202].

[29] A. U. Abeysekara et al., Astropart. Phys. 35, 641 (2012).

[30] G. S. Bowers, D. M. Smith, G. F. Martinez-McKinney, M. Kamogawa, S. A. Cummer, J. R. Dwyer, D. Wang, M. Stock, and Z. Kawasaki, Geophys. Res. Lett. 44, 10 (2017).

[31] Y. Wada, G. S. Bowers, T. Enoto, M. Kamogawa, Y. Nakamura, T. Morimoto, D. M. Smith, Y. Furuta, K. Nakazawa, T. Yuasa, A. Matsuki, M. Kubo, T. Tamagawa, 
K. Makishima, and H. Tsuchiya, Geophys. Res. Lett. 45, 5700 (2018).

[32] See Supplemental Material at http://link.aps.org/ supplemental/10.1103/PhysRevD.100.043021 for detailed descriptions of the instrument response simulations, as well as the background modeling, modification of cosmic-ray background spectra (MOS) by thunderstorm electric field modeling, and the point-source relativistic runaway electron avalanche (RREA) modeling. We also describe how the uncertainties were calculated for these results.

[33] S. Agostinelli et al. (GEANT4 Collaboration), Nucl. Instrum. Methods Phys. Res., Sect. A 506, 250 (2003).

[34] J. Allison et al., IEEE Trans. Nucl. Sci. 53, 270 (2006).

[35] J. Allison et al., Nucl. Instrum. Methods Phys. Res., Sect. A 835, 186 (2016).

[36] D. Sarria, C. Rutjes, G. Diniz, A. Luque, K. M. A. Ihaddadene, J. R. Dwyer, N. Østgaard, A. B. Skeltved,
I. S. Ferreira, and U. Ebert, Geosci. Model Dev. 11, 4515 (2018).

[37] T. Sato, PLoS One 10, 1 (2015).

[38] T. Sato, PLoS One 11, 1 (2016).

[39] The U.S Standard Atmosphere (U.S. Government Printing Office, 1976).

[40] X.-M. Shao, C. Ho, M. Caffrey, P. Graham, B. Haynes, G. Bowers, W. Blaine, B. Dingus, D. Smith, and H. Rassoul, J. Geophys. Res. 123, 10 (2018).

[41] B. J. Hazelton, B. W. Grefenstette, D. M. Smith, J. R. Dwyer, X.-M. Shao, S. A. Cummer, T. Chronis, E. H. Lay, and R. H. Holzworth, Geophys. Res. Lett. 36, L01108 (2009).

[42] T. C. Marshall and M. Stolzenburg, J. Geophys. Res. 106, 4757 (2001).

[43] B. Hariharan et al. (GRAPES-3 Collaboration), Phys. Rev. Lett. 122, 105101 (2019).

[44] J. R. Dwyer, Phys. Plasmas 14, 042901 (2007).

[45] A. Chilingarian, Phys. Rev. D 98, 022007 (2018). 